\title{
Quantitative analysis of polydioxanone thread effects on lid-cheek junction
}

\author{
Uekyoung Hwang, MD, MPH${ }^{1}$, Juyoung Lee, MD¹, Kyoungjin Kang, MD, PhD² \\ ${ }^{1}$ Yeline Esthetic Clinic, Seongnam, Rep. of Korea, ${ }^{2}$ Seoul Cosmetic Surgery Clinic, Busan, Rep. of Korea
}

\begin{abstract}
Background: Polydioxanone (PDO) thread is commonly used as a skin rejuvenation material in the field of cosmetic medicine. Its usefulness in the aged eye area was described previously, but, to date, no clinical study has proven it.

Objective: The purpose of this study was to investigate the rejuvenation effects of PDO thread on the aged lower eye area thorough quantitative and qualitative analysis.

Methods: We examined the changes in the lid-cheek junction in 32 participants who underwent the PDO thread insertion. They were followed up during 12 weeks after PDO thread insertion into the orbital fat area. Participants were categorized into three groups by tear trough grade; the eyelid to junction distance (EJD)/eyebrow to junction distance (BJD) ratio was measured before and after the PDO thread insertion. Differences in EJD to BJD ratio after PDO thread insertion and post-procedural differences in EJD to BJD ratio by tear trough grade were compared by the paired t-test.

Results: After PDO insertion into the infraorbital fat pad area, the overall EJD to BJD ratio significantly decreased from $0.35 \pm 0.03$ to $0.33 \pm 0.04$ ( $p=0.001$ ). The significant post-procedural improvements in EJD to BJD ratio were found in grade 1 group and grade 2 group: $0.35 \pm 0.03$ to $0.34 \pm 0.04$ for grade 1 group $(p=0.02$ ), and $0.34 \pm 0.04$ to $0.32 \pm 0.04$ for grade 2 group ( $p=0.03$ ); however, the changes were not found in the grade 3 group $(p=0.21)$.

Conclusion: PDO thread insertion into the aged lower eyelid may be an effective method for improving signs of lower aged eyelid particularly in individuals with tear trough group 1 and group 2.
\end{abstract}

Keywords: EJD/BJD; infraorbital fat protrusion; lid-cheek junction; polydioxanone; rejuvenation

\section{Introduction}

The aged lower eyelid is typically characterized by skin laxity with wrinkles, protruding orbital fat, and tear trough deformity [1], all of which are potentially caused by aging processes such as bone resorption, attenuation of the retaining ligament and septum, decreases in collagen deposition in the skin and subcutaneous layer, and descending suborbicularis oculi fat $[2,3]$.

As previously documented in a case report [4], polydioxanone (PDO) thread insertion showed the potential effect of flattening of the protruding orbital fat, skin tightening, wrinkle improvement, volume restoration in tear trough deformity, and skin whitening.

For improving the appearance of aged lower eyelids, understanding the changes in anatomical structures between the lower lid and midface is important from a cosmetic medicine perspective. Therefore, the identification of changes at the lidcheek junction (LCJ) [5], which is located between the lower lid and midface, may be useful for preoperatively classifying aging degree and postoperatively evaluating rejuvenation effects.

Received February 5, 2018; Revised None; Accepted February 17, 2018

Corresponding author: Uekyoung Hwang

E-mail: yjukhome@daum.net, ORCID: https://orcid.org/0000-0002-0923-9830

This is an Open Access article distributed under the terms of the Creative Commons Attribution Non-Commercial License (http://creativecommons.org/licenses/by-nc/4.0), which permits unrestricted non-commercial use, distribution, and reproduction in any medium, provided the original work is properly cited.

Copyright ( 2018 Korean Society of Korean Cosmetic Surgery and Medicine (KSKCS \& KCCS). 
The LCJ in young people has a gentle and round curve and is located at or below the infraorbital rim. However, with aging, it changes to a $\mathrm{V}$ shape and is always located below the infraorbital rim. In a previous study of a Western population, the vertical distance from the upper edge of the lower lid to the LCJ in youthful eyes is 8 to $12 \mathrm{~mm}$ and it elongates to 15 to18 $\mathrm{mm}$ in aged eyes [6]. The purpose of this study was to investigate the rejuvenation effects of PDO thread on the aged lower eye area thorough quantitative and qualitative analysis using LCJ distance.

\section{Materials and methods}

\section{Study participants}

Between 2017 May and 2017 December, a total 34 participants (aged 22-68 years; 33 women, one man; none had undergone any eye procedures in the previous 5 years) volunteered to undergo PDO thread insertion in the area of the infraorbital fat

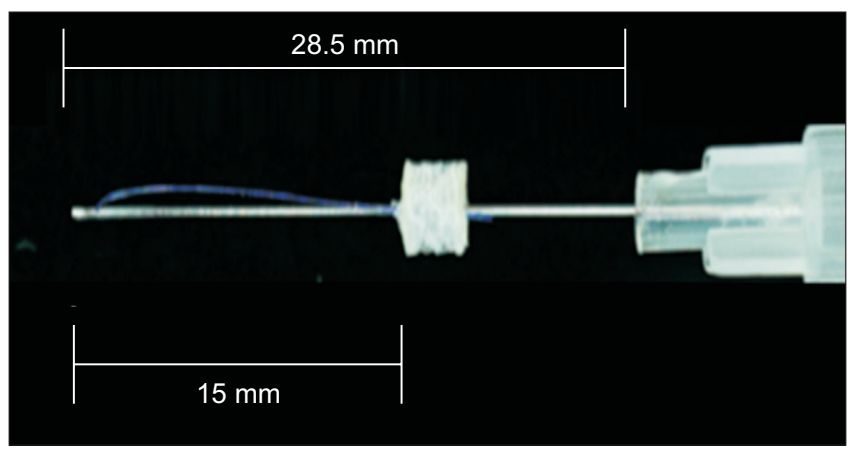

Fig. 1. Schematic figure of the polydioxanone (PDO) thread device (Klothread Eye Suture Kit; Meditronic Dongjun, Jeonju, Korea). A 28.5-mm length of PDO thread (6.0 USP monofilament) was inserted into the cannula (30 gauge; $28.5 \mathrm{~mm}$ in length), with 15 $\mathrm{mm}$ located inside the cannula and $15 \mathrm{~mm}$ placed on its surface.

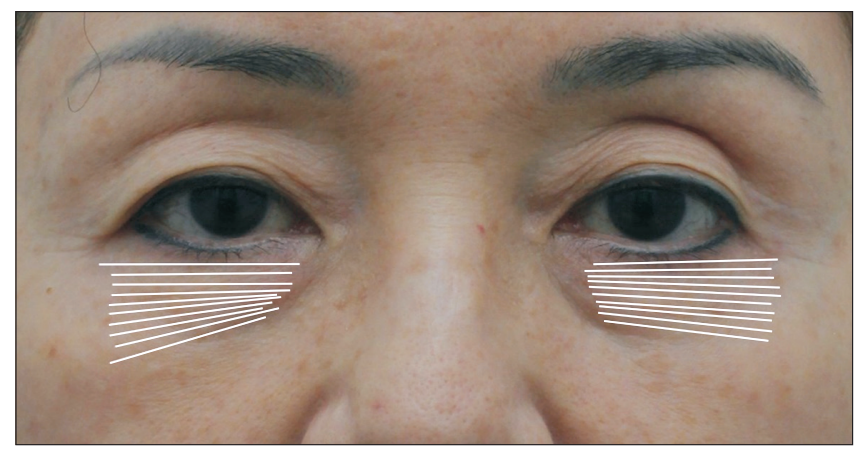

Fig. 2. Schematic view of insertion points for the polydioxanone (PDO) threads. The threads were inserted into the submuscular layer, protruding orbital fat pad, and orbital retaining ligament. The white arrows indicate the direction and placement of the PDO threads. pad and attended 12 weeks of follow-up visits. Two participants were lost to follow-up and 32 participants were included in the final analysis.

\section{PDO thread device and PDO thread insertion protocol}

A $30 \mathrm{~mm}$ length of PDO thread (6.0 USP monofilament, Klothread Eye Suture Kit; Meditronic Dongjun, Jeonju, Korea) was inserted into a cannula (30 gauge; $28.5 \mathrm{~mm}$ in length). Of the $30 \mathrm{~mm}$ length of PDO thread, $15 \mathrm{~mm}$ was located inside and $15 \mathrm{~mm}$ was placed on the cannula surface (Fig. 1). Local anesthesia using a $2 \%$ lidocaine solution with epinephrine (1:100,000) was administered to the entry sites and the protruding orbital fat pad. Ten PDO threads were advanced through the submuscular layer and inserted into the protruding orbital fat pad and orbital retaining ligament at 1-mm intervals into the lower eye area of the patient as depicted in Fig. 2. During the thread insertion, the upper eyelid was maximally opened upward to keep the orbital fat pad prominently displayed. Following thread insertion, the lower eyelid was compressed with gauze and 3M tape (Scotch, Maplewood, MN, USA) was applied for 1 day to prevent swelling and further bleeding.

\section{Measuring LCJ change: eyelid to junction distance to brow to junction distance ratio}

We measured the distances from the lower eyelid margin to the LCJ (eyelid to junction distance, EJD) and from the eyebrow to the LCJ (BJD). The ratios of EJD to BJD (EJD/BJD) were calculated by mean values on the both sides and compared between the pre- and post-procedure photographs (Fig. 3). According to Hermand's tear trough grading system [7], the participants were classified into groups 1 (mild tear trough), group 2 (moderate tear trough), and group 3 (severe tear trough). Grade 1 included

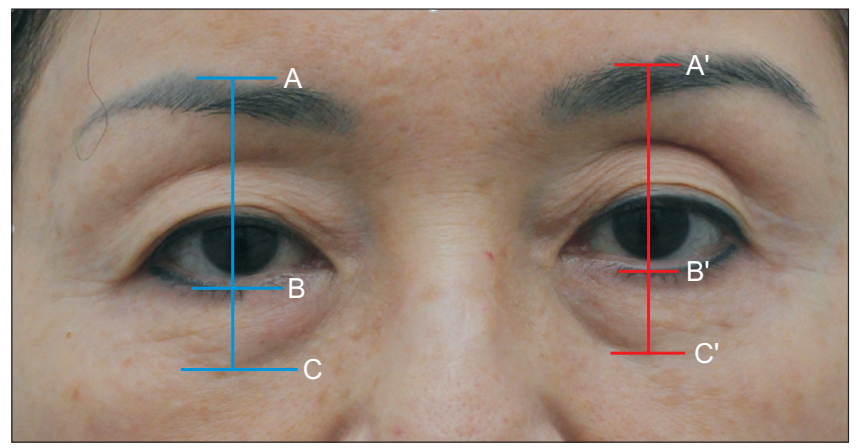

Fig. 3. Measurement ratio of lower eyelid to cheek junction distance (EJD) and that of eyebrow to junction distance (BJD). $\mathrm{AC}\left(\mathrm{A}^{\prime} \mathrm{C}^{\prime}\right)$ line drawn vertically from the center of the pupil. $A C\left(\mathrm{~A}^{\prime} \mathrm{C}^{\prime}\right)$; $\mathrm{BJD}, \mathrm{BC}$ $\left(\mathrm{B}^{\prime} \mathrm{C}^{\prime}\right) ; \mathrm{EJC}, \mathrm{EJD} / \mathrm{BJD}=\mathrm{BC}\left(\mathrm{B}^{\prime} \mathrm{C}^{\prime}\right) / \mathrm{AC}\left(\mathrm{A}^{\prime} \mathrm{C}^{\prime}\right)$. 
those with hollowness medially and above the infraorbital rim, grade 2 included those with a hollow curve below the infraorbital rim and/or laterally beyond the pupil, and grade 3 included those with a completely visible infraorbital rim.

\section{Statistical analysis}

Differences in EJD to BJD ratio after PDO thread insertion and post-procedural differences in EJD to BJD ratio by tear trough grade were compared by the paired t-test. All analysis were conducted using SAS statistical software (version 9.4; SAS Institute Inc., Cary, NC, USA). All statistical tests were two-sided, and statistical significance was determined at $\mathrm{p}<0.05$.

\section{Results}

\section{Quantitative results}

Baseline characteristics of the study population are presented in Table 1. Our study included 31 women and 1 man with a mean age of $46.6 \pm 11.7$ years old and the follow-up period was 12 weeks post-procedure. Tear trough grade was categorized into three groups and the grade 2 was most prevalent (53.1\%).

Overall change in EJD to BJD ratio is shown in Table 2. Before the procedure, the mean EJD to BJD ratio was 0.35 \pm 0.03 ; after PDO insertion into the infraorbital fat pad area, the mean ratio was $0.33 \pm 0.04(\mathrm{p}=0.001)$.

Table 3 shows post-procedural changes in EJD to BJD ratios

Table 1. Baseline characteristics of the study population $(n=32)$

\begin{tabular}{|cc|}
\hline \multicolumn{1}{c}{ Variable } & Value \\
\hline Age $(\mathrm{yr})$ & $46.6 \pm 11.7$ \\
\hline Sex & $31(96.9)$ \\
\hline Female & $1(3.1)$ \\
\hline Male & \\
\hline Tear trough grade & $7(21.9)$ \\
\hline Grade 1 & $17(53.1)$ \\
\hline Grade 2 & $8(25.0)$ \\
\hline Grade 3
\end{tabular}

Values are presented as mean \pm standard deviation or number (\%). by tear trough grade. The significant post-procedural improvements in EJD to BJD ratio were found in group 1 and grade 2 group: $0.35 \pm 0.03$ to $0.34 \pm 0.04$ for grade 1 group $(\mathrm{p}=0.02)$, and $0.34 \pm 0.04$ to $0.32 \pm 0.04$ for grade 2 group $(\mathrm{p}=0.03$ ); however, the changes were not found in the grade 3 group $(\mathrm{p}=0.21)$.

\section{Qualitative morphological results}

Morphologic changes (infraorbital fat bulge, fine wrinkles, and periorbital pigmentation and color change) before versus after the procedure were also observed using photography. After the PDO thread insertion, infraorbital fat bulging, fine wrinkles, and periorbital pigmentation and color was improved with significant EJD/BJD ratio changes in group 1 and group 2 (Fig. 4, 5). These morphological changes were also observed in group 3, but there was no statistical significance (Fig. 6).

\section{Discussion}

In this study, we found the EJD/BJD ratios after PDO thread insertion significantly decreased in the tear trough grade 1 and grade 2 group but not in the tear trough grade 3 group. In a prior preliminary study [4], we reported the potential effects of PDO thread insertion on the improvement in orbital fat protrusion, likely due to contraction of the fat layer accompanied by fibrotic band formation in the subcutaneous fat layer. In the current study, EJD/BJD ratio decreased after the PDO insertion into the protruding orbital fat consistent with previously published results [8]. Our findings suggest that PDO thread insertion into the aged lower eyelid may be an effective method for improv-

Table 2. Overall changes in EJD to BJD ratio after polydioxanone thread insertion

\begin{tabular}{|c|c|c|c|}
\hline \multicolumn{2}{|c|}{ EJD to BJD ratio } & \multirow{2}{*}{$\begin{array}{l}\text { Difference in EJD to } \\
\text { BJD after procedure }\end{array}$} & \multirow{2}{*}{ p-value } \\
\hline Before & After & & \\
\hline $0.35 \pm 0.03$ & $0.33 \pm 0.04$ & $-0.02 \pm 0.02$ & 0.001 \\
\hline
\end{tabular}

Values are presented as mean \pm standard deviation. The p-value was calculated by paired t-test.

EJD, eyelid to junctional distance; BJD, eyebrow to junctional distance.

Table 3. Post-procedural changes in EJD to BJD ratios by tear trough grade

\begin{tabular}{lcccc}
\hline \multirow{2}{*}{ Grade } & \multicolumn{2}{c}{ EJD to BJD ratio } & & $\begin{array}{c}\text { Difference in EJD to BJD after } \\
\text { procedure }\end{array}$ \\
\cline { 2 - 5 } & Before & After & $-0.02 \pm 0.01$ & p-value \\
\hline Grade $1(\mathrm{n}=7)$ & $0.35 \pm 0.03$ & $0.34 \pm 0.04$ & $-0.02 \pm 0.02$ & 0.02 \\
Grade 2 $(\mathrm{n}=17)$ & $0.34 \pm 0.04$ & $0.32 \pm 0.04$ & $-0.01 \pm 0.01$ & 0.03 \\
Grade 3 $(\mathrm{n}=8)$ & $0.35 \pm 0.03$ & $0.34 \pm 0.04$ & 0.21 \\
\hline
\end{tabular}

Values are presented as mean \pm standard deviation. The $\mathrm{p}$-value was calculated by paired $\mathrm{t}$-test.

EJD, eyelid to junctional distance; BJD, eyebrow to junctional distance. 

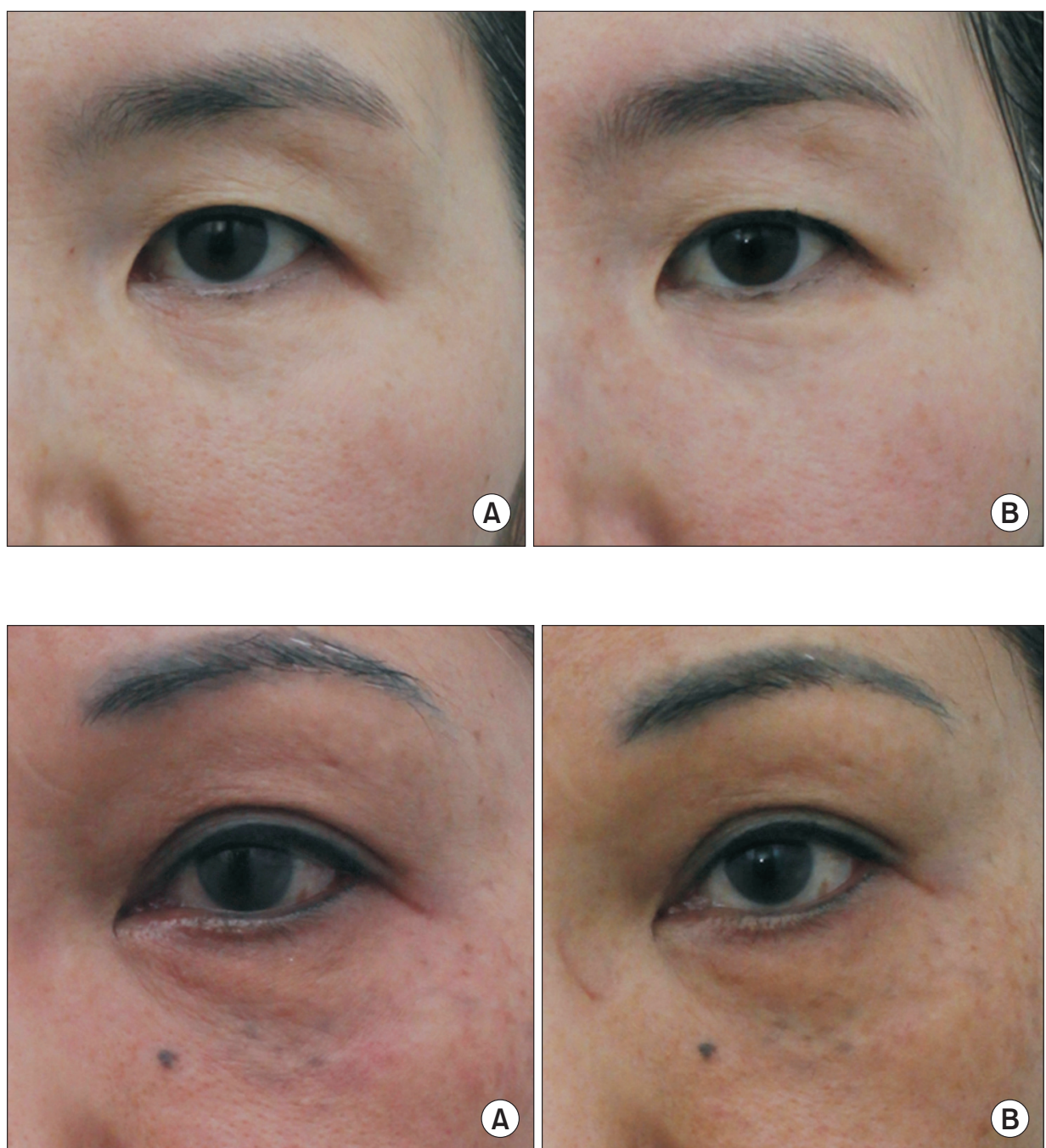

Fig. 5. Lid-cheek junction change of tear trough grade 2. (A) Before polydioxanone (PDO) insertion. (B) After PDO insertion.
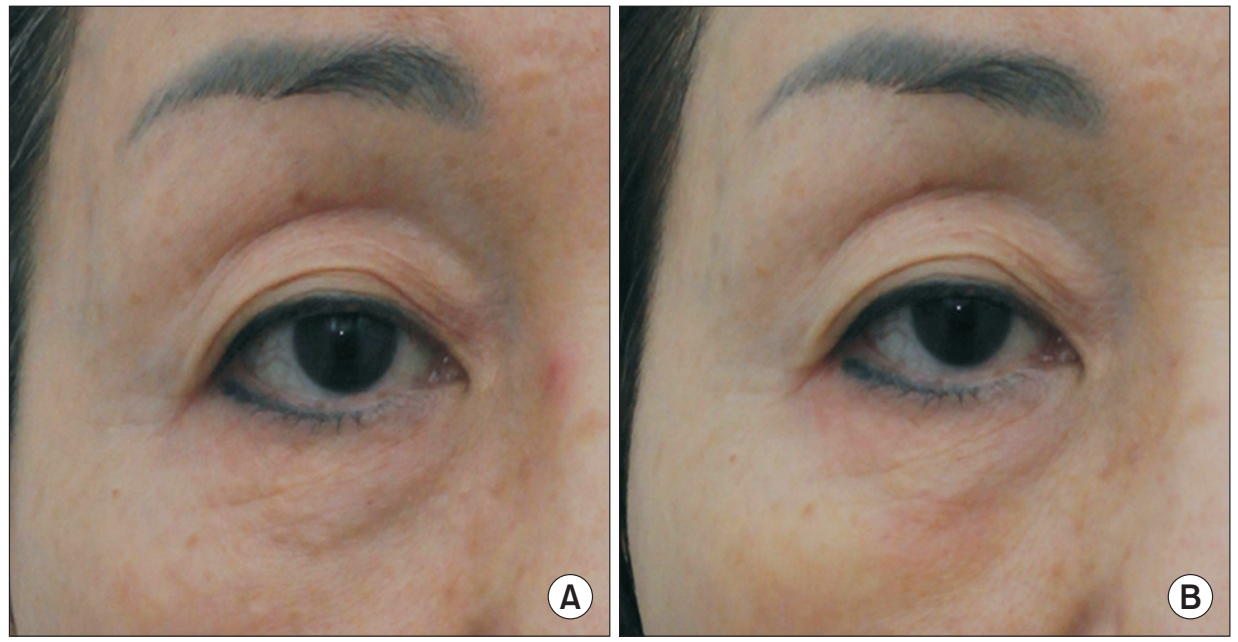

Fig. 4. Lid-cheek junction change of tear trough grade 1. (A) Before the polydioxanone (PDO) insertion. (B) After PDO insertion.
Fig. 6. Lid-cheek junction change of tear trough grade 3. (A) Before polydioxanone (PDO) insertion. (B) After PDO insertion. 
ing signs of lower eyelid aging particularly in individuals with tear trough grade 1 and 2 group. The PDO thread insertion procedure is a minimally invasive and easy technique that has a tissue rejuvenation effect; however, in severe case, it may not correct the anatomical defect.

Orbital fat protrusion, skin laxity with wrinkles, tear trough deformity, and hyperpigmentation are typical characteristics of the aged lower eyelid. Various esthetic procedures have been developed to improve the appearance of the aged lower eyelid. The tear trough can be improved by decreases in orbital fat protrusion, increased tightening and thickening of the orbital retaining ligament, and decreases in skin laxity. Inserted PDO thread may serve as a tissue scaffold. Mechanistically, this scaffold acts as an activator of intercellular interactions, collagen deposition, neovascularization, and mechanotransduction [9]. The mechanotransductive signaling in fibroblasts produces cytoskeletal remodeling following acupuncture to the subcutaneous fat layer [10]. The fibrotic encapsulation of the PDO threads following insertion into the fat layer can potentially lead to contraction of the subcutaneous fat layer [11]; thus, the eyelidcheek delineation becomes more pronounced. Moreover, the disappearance of eyelid wrinkling following PDO stimulation was likely attributable to increased collagen synthesis and activation of cell-cell interactions. Biomechanical stimulation following PDO biodegradation in the tissues further caused enhanced whitening of the skin color, likely caused by both neovascularization and increased cellular transforming growth factor- $\beta$ signaling [12], which has been demonstrated as an important inhibitor of melanogenesis [13].

In conclusion, PDO thread insertion into the aged lower eyelid may be an effective method for improving signs of lower eyelid aging particularly in individuals with tear trough group 1 and group 2. Additional long-term observations are warranted to demonstrate the safety of this treatment methodology.

\section{Conflicts of interest}

The authors have nothing to disclose.

\section{References}

1. Farkas JP, Pessa JE, Hubbard B, Rohrich RJ. The science and theory behind facial aging. Plast Reconstr Surg Glob Open 2013;1:e8-15.

2. Levesque AY, de la Torre JI. Midface anatomy, aging, and aesthetic analysis. Facial Plast Surg Clin North Am 2015;23:12936.

3. Friedmann DP, Goldman MP. Dark circles: etiology and management options. Clin Plast Surg 2015;42:33-50.

4. Hwang UK, Kwon YH, Kang KJ. Improvement of aged lower eyelid by insertion of polydioxanone threads. J Cosme Med 2017;1:57-9.

5. Lambros V. Observations on periorbital and midface aging. Plast Reconstr Surg 2007;120:1367-76; discussion 1377.

6. Hester TR Jr, Codner MA, McCord CD, Nahai F, Giannopoulos A. Evolution of technique of the direct transblepharoplasty approach for the correction of lower lid and midfacial aging: maximizing results and minimizing complications in a 5-year experience. Plast Reconstr Surg 2000;105:393-406; discussion 407-8.

7. Hirmand H. Anatomy and nonsurgical correction of the tear trough deformity. Plast Reconstr Surg 2010;125:699-708.

8. Kang KJ, Yang HH, Chai CY. Assessment of rejuvenation by change of lid-cheek junction after transconjunctival and traditional subciliary incision blepharoplasty. J Cosmet Med 2017;1:30-8.

9. Yoon JH, Kim SS, Kim DI. Skin rejuvenation to make use of absorbable PDO suture in regenerative medicine. Seoul: MD WORLD Medical Publishing Co.; 2012.

10. Langevin HM, Bouffard NA, Badger GJ, Churchill DL, Howe AK. Subcutaneous tissue fibroblast cytoskeletal remodeling induced by acupuncture: evidence for a mechanotransduction-based mechanism. J Cell Physiol 2006;207:767-74.

11. Gerarduzzi C, Di Battista JA. Myofibroblast repair mechanisms post-inflammatory response: a fibrotic perspective. Inflamm Res 2017;66:451-65.

12. Kim J, Zheng Z, Kim H, Nam KA, Chung KY. Investigation on the cutaneous change induced by face-lifting monodirectional barbed polydioxanone thread. Dermatol Surg 2017;43:7480.

13. Kim WS, Park SH, Ahn SJ, Kim HK, Park JS, Lee GY, et al. Whitening effect of adipose-derived stem cells: a critical role of TGF-beta 1. Biol Pharm Bull 2008;31:606-10. 